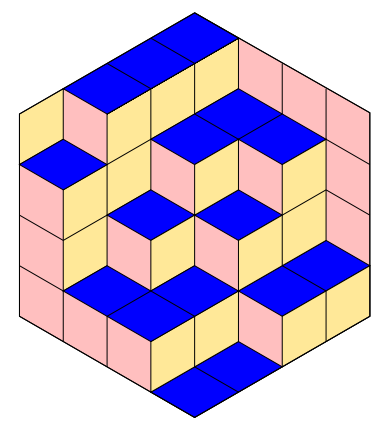

Sebastian M. Cioabă, Jack H. Koolen \& Hiroshi Nozaki

A spectral version of the Moore problem for bipartite regular graphs

Volume 2, issue 6 (2019), p. 1219-1238.

<http://alco.centre-mersenne.org/item/ALCO_2019__2_6_1219_0>

(c) The journal and the authors, 2019.

Some rights reserved.

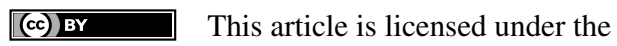

Creative Commons ATtribution 4.0 International LiCEnSE.

http://creativecommons.org/licenses/by/4.0/

Access to articles published by the journal Algebraic Combinatorics on the website http://alco.centre-mersenne.org/ implies agreement with the Terms of Use (http://alco.centre-mersenne.org/legal/).

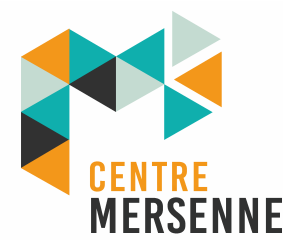

Algebraic Combinatorics is member of the Centre Mersenne for Open Scientific Publishing www.centre-mersenne.org 


\title{
A spectral version of the Moore problem for bipartite regular graphs
}

\author{
Sebastian M. Cioabă, Jack H. Koolen \& Hiroshi Nozaki
}

\begin{abstract}
Let $b(k, \theta)$ be the maximum order of a connected bipartite $k$-regular graph whose second largest eigenvalue is at most $\theta$. In this paper, we obtain a general upper bound for $b(k, \theta)$ for any $0 \leqslant \theta<2 \sqrt{k-1}$. Our bound gives the exact value of $b(k, \theta)$ whenever there exists a bipartite distance-regular graph of degree $k$, second largest eigenvalue $\theta$, diameter $d$ and girth $g$ such that $g \geqslant 2 d-2$. For certain values of $d$, there are infinitely many such graphs of various valencies $k$. However, for $d=11$ or $d \geqslant 15$, we prove that there are no bipartite distance-regular graphs with $g \geqslant 2 d-2$.
\end{abstract}

\section{INTRODUCTION}

Let $\Gamma=(V, E)$ be a connected $k$-regular simple graph with $n$ vertices. For $1 \leqslant i \leqslant n$, let $\lambda_{i}(\Gamma)$ denote the $i$-th largest eigenvalue of the adjacency matrix of $\Gamma$. The eigenvalues have close relationships with other graph invariants. The smallest eigenvalue $\lambda_{n}(\Gamma)$ is related to the diameter, the chromatic number and the independence number (see [8, Chapter 4] or [9] for example). The second eigenvalue $\lambda_{2}(\Gamma)$ plays a fundamental role in the study of expanders $[2,3,8,20]$. Let $v(k, \theta)$ denote the maximum order of a connected $k$-regular graph $\Gamma$ with $\lambda_{2}(\Gamma) \leqslant \theta$. For $\theta<2 \sqrt{k-1}$, from work of Alon and Boppana, and Serre, we know that the value $v(k, \theta)$ is finite (see [10, 25]). In [10], we obtained the following upper bound for $v(k, \theta)$. Let $\boldsymbol{T}(k, t, c)$ be the $t \times t$ tridiagonal matrix with lower diagonal $(1,1, \ldots, 1, c)$, upper diagonal $(k, k-1, \ldots, k-1)$, and with constant row sum $k$. If $\theta$ is the second largest eigenvalue of $\boldsymbol{T}(k, t, c)$, then

$$
v(k, \theta) \leqslant 1+\sum_{i=0}^{t-3} k(k-1)^{i}+\frac{k(k-1)^{t-2}}{c} .
$$

Equality holds in (1) if and only if there is a distance-regular graph of valency $k$ with second largest eigenvalue $\theta$, girth $g$ and diameter $d$ satisfying $g \geqslant 2 d$. For $d>6$, there are no such graphs [12]. However, for smaller values of $d$, there are infinitely many values of $k$ and $\theta$ where the above inequality gives the exact value of $v(k, \theta)$.

Manuscript received 2nd May 2018, revised and accepted 4th March 2019.

KEYWORDS. second eigenvalue, bipartite regular graph, bipartite distance-regular graph, expander, linear programming bound.

ACKnowledgements. Sebastian M. Cioabă is supported by NSF grants DMS-1600768 and CIF1815922. Jack H. Koolen is partially supported by the National Natural Science Foundation of China (Nos. 11471009 and 11671376). He also acknowledges the financial support of the Chinese Academy of Sciences under its "100 talent" program. Hiroshi Nozaki is partially supported by JSPS Grants-in-Aid for Scientific Research Nos. 16K17569, 17K05155, and 18K03396. 
In this paper, we improve the above results from [10] for bipartite regular graphs. Let $b(k, \theta)$ denote the maximum order of a connected bipartite $k$-regular graph $\Gamma$ with $\lambda_{2}(\Gamma) \leqslant \theta$. Bipartite regular graphs $\Gamma$ with $\lambda_{2}(\Gamma) \leqslant \theta$ have been classified for $\theta=\sqrt{2}[29], \theta=\sqrt{3}[22]$, and $\theta=2$ [23]. We obtain a general upper bound for $b(k, \theta)$ for any $0 \leqslant \theta<2 \sqrt{k-1}$. Our bound gives the exact value of $b(k, \theta)$ whenever there exists a bipartite distance-regular graph of degree $k$ with second largest eigenvalue $\theta$, diameter $d$ and girth $g$ such that $g \geqslant 2 d-2$. For certain values of $d$, there are infinitely many such graphs of various valencies $k$. When $d=11$ or $d \geqslant 15$, we prove the non-existence of bipartite distance-regular graphs with $g \geqslant 2 d-2$. Our results generalize previous work of Høholdt and Justesen [19] obtained in their study of graph codes and imply some results of Li and Solé [24] relating the second largest eigenvalue of a bipartite regular graph to its girth. The degree-diameter or Moore problem for graphs [26] is about determining the largest graphs of given maximum degree and diameter. Given the connections between the diameter and the second largest eigenvalue of bipartite regular graphs (see [9] for example), our Theorem 4.1 can be interpreted as a spectral version of the Moore problem for bipartite regular graphs.

In Section 2, we describe some sequences of orthogonal polynomials and develop the preliminary results and notation that will be used in the paper. In Section 3, we improve the linear programming bound from [27] for the class of bipartite regular graphs. In Section 4, we obtain the following upper bound for $b(k, \theta)$. Let $\boldsymbol{B}(k, t, c)$ be the $t \times t$ tridiagonal matrix with lower diagonal $(1, \ldots, 1, c, k)$, upper diagonal $(k, k-1, \ldots, k-1, k-c)$, and constant row sum $k$. If $\theta$ is the second largest eigenvalue of $\boldsymbol{B}(k, t, c)$, then

$$
b(k, \theta) \leqslant 2\left(\sum_{i=0}^{t-4}(k-1)^{i}+\frac{(k-1)^{t-3}}{c}+\frac{(k-1)^{t-2}}{c}\right) .
$$

We show that equality happens in (2) when there is a bipartite distance-regular graph of degree $k$, second largest eigenvalue $\theta$ having $g \geqslant 2 d-2$. Inequality (2) generalizes some results of Høholdt and Justesen [19] (see Corollaries 4.8 and 4.9), and of Li and Solé [24] (see Corollary 4.11). At the end of Section 4, we prove that the bound (2) is better than (1) for any $k$ and $\theta$. In Section 5 , we prove the non-existence of bipartite distance-regular graphs with $g \geqslant 2 d-2$ for $d=11$ and $d \geqslant 15$. We conclude the paper with some remarks in Section 6.

\section{Preliminaries}

In this section, we describe some useful polynomials that will be used to prove our main result. For any integer $k \geqslant 2$, let $\left(F_{i}^{(k)}\right)_{i \geqslant 0}$ be a sequence of orthogonal polynomials defined by the three-term recurrence relation:

$$
F_{0}^{(k)}(x)=1, \quad F_{1}^{(k)}(x)=x, \quad F_{2}^{(k)}(x)=x^{2}-k,
$$

and

$$
F_{i}^{(k)}(x)=x F_{i-1}^{(k)}(x)-(k-1) F_{i-2}^{(k)}(x)
$$

for $i \geqslant 3$. The notation $F_{i}^{(k)}$ is abbreviated to $F_{i}$ for the rest of the paper. Let $q=\sqrt{k-1}$. The polynomials $\left(F_{i}\right)_{i \geqslant 0}$ form a sequence of orthogonal polynomials with respect to the positive weight

$$
w(x)=\frac{\sqrt{4 q^{2}-x^{2}}}{k^{2}-x^{2}}
$$


on the interval $[-2 q, 2 q]$ (see $[21$, Section 4$]$ ). The polynomials $F_{i}(q y) / q^{i}$ in $y$ are called Geronimus polynomials $[16,17]$. It follows from $(3)$ that

$$
F_{i}(x)=\left(x^{2}-2 k+2\right) F_{i-2}(x)-(k-1)^{2} F_{i-4}(x)
$$

for $i \geqslant 5$. Note that for any $i \geqslant 0, F_{2 i}(x)$ and $F_{2 i+1}(x)$ are even and odd functions of $x$, respectively.

For $i \geqslant 0$, let $\mathscr{F}_{0, i}(x)=F_{2 i}(\sqrt{x})$ and $\mathscr{F}_{1, i}(x)=F_{2 i+1}(\sqrt{x}) / \sqrt{x}$. It follows that $x^{\epsilon} \mathscr{F}_{\epsilon, i}\left(x^{2}\right)=F_{2 i+\epsilon}(x)$ for $\epsilon \in\{0,1\}$. By $(4)$, the polynomials $\mathscr{F}_{0, i}(x)$ and $\mathscr{F}_{1, i}(x)$ satisfy the following properties:

$$
\begin{aligned}
\mathscr{F}_{0,0}(x)=1, \quad \mathscr{F}_{0,1}(x) & =x-k, \quad \mathscr{F}_{0,2}(x)=x^{2}-(3 k-2) x+k(k-1), \\
\mathscr{F}_{1,0}(x) & =1, \quad \mathscr{F}_{1,1}(x)=x-(2 k-1),
\end{aligned}
$$

and

$$
\mathscr{F}_{\epsilon, i}(x)=(x-2 k+2) \mathscr{F}_{\epsilon, i-1}(x)-(k-1)^{2} \mathscr{F}_{\epsilon, i-2}(x)
$$

for any $i \geqslant 3$ if $\epsilon=0$, and $i \geqslant 2$ if $\epsilon=1$. Note that $k^{\epsilon} \mathscr{F}_{\epsilon, i}\left(k^{2}\right)=F_{2 i+\epsilon}(k)=$ $k(k-1)^{2 i-1+\epsilon}=(k-1)^{2 i-1+\epsilon}+(k-1)^{2 i+\epsilon}$ for $2 i+\epsilon \neq 0$. For $\epsilon \in\{0,1\}$, the polynomials $\left(\mathscr{F}_{\epsilon, i}\right)_{i \geqslant 0}$ form a sequence of orthogonal polynomials with respect to the positive weight

$$
w_{\epsilon}(x)=\frac{x^{\epsilon-1 / 2} \sqrt{4 q^{2}-x}}{k^{2}-x}
$$

on the interval $\left[0,4 q^{2}\right]$.

For $i \geqslant 0$, let $G_{i}(x)=\sum_{j=0}^{\lfloor i / 2\rfloor} F_{i-2 j}(x)$. A simple calculation implies that

$$
G_{i}(x)=\frac{F_{i+2}(x)-(k-1)^{2} F_{i}(x)}{x^{2}-k^{2}}
$$

for $i \geqslant 1$. From Lemmas 3.3 and 3.5 in [11], the polynomials $\left(G_{i}\right)_{i \geqslant 0}$ form a sequence of orthogonal polynomials with respect to the positive weight $\left(k^{2}-x^{2}\right) w(x)=\sqrt{4 q^{2}-x^{2}}$ on the interval $[-2 q, 2 q]$. From (3), we deduce that

$$
G_{i}(x)=x G_{i-1}(x)-(k-1) G_{i-2}(x)
$$

for $i \geqslant 2$.

Let $\mathscr{G}_{\epsilon, i}(x)$ denote the polynomial

$$
\mathscr{G}_{\epsilon, i}(x)=\sum_{j=0}^{i} \mathscr{F}_{\epsilon, j}(x) .
$$

It follows that $x^{\epsilon} \mathscr{G}_{\epsilon, j}\left(x^{2}\right)=G_{2 j+\epsilon}(x)$. Using (6), the polynomial $\mathscr{G}_{\epsilon, i}(x)$ can be expressed as

$$
\mathscr{G}_{\epsilon, i}(x)=\frac{\mathscr{F}_{\epsilon, i+1}(x)-(k-1)^{2} \mathscr{F}_{\epsilon, i}(x)}{x-k^{2}}
$$

for any $i \geqslant 2$ if $\epsilon=0$, and $i \geqslant 1$ if $\epsilon=1$. From Lemmas 3.3 and 3.5 in [11], for $\epsilon \in\{0,1\}$, the polynomials $\left(\mathscr{G}_{\epsilon, i}\right)_{i \geqslant 0}$ form a sequence of orthogonal polynomials with respect to the positive weight $\left(k^{2}-x\right) w_{\epsilon}(x)=x^{\epsilon-1 / 2} \sqrt{4 q^{2}-x}$ on the interval $\left[0,4 q^{2}\right]$.

LEMMA 2.1. Let $p_{l}(i, j)$ be the coefficients in $x^{\epsilon} \mathscr{F}_{\epsilon, i}(x) \mathscr{F}_{\epsilon, j}(x)=\sum_{l=0}^{i+j+\epsilon} p_{l}(i, j) \mathscr{F}_{0, l}(x)$. Then we have $p_{0}(i, j)=k^{\epsilon} \mathscr{F}_{\epsilon, i}\left(k^{2}\right) \delta_{i, j}$, and $p_{l}(i, j) \geqslant 0$ for any $l, i, j$. Moreover $p_{l}(i, j)>0$ if and only if $|i-j| \leqslant l \leqslant i+j+\epsilon$. 
Proof. We have that

$$
F_{2 i+\epsilon}(x) F_{2 j+\epsilon}(x)=x^{2 \epsilon} \mathscr{F}_{\epsilon, i}\left(x^{2}\right) \mathscr{F}_{\epsilon, j}\left(x^{2}\right)=\sum_{l=0}^{i+j+\epsilon} p_{l}(i, j) \mathscr{F}_{0, l}\left(x^{2}\right)=\sum_{l=0}^{i+j+\epsilon} p_{l}(i, j) F_{2 l}(x) .
$$

By Theorem 3 in [27], we obtain that $p_{0}(i, j)=F_{2 i+\epsilon}(k) \delta_{i, j}=k^{\epsilon} \mathscr{F}_{\epsilon, i}\left(k^{2}\right) \delta_{i, j}$, and $p_{l}(i, j) \geqslant 0$ for any $l, i, j$. Moreover $p_{l}(i, j)>0$ if and only if $|i-j| \leqslant l \leqslant i+j+\epsilon$.

Let $\Gamma$ be a connected regular bipartite graph. The adjacency matrix $\boldsymbol{A}$ of $\Gamma$ can be expressed by

$$
A=\left(\begin{array}{cc}
O & N \\
N^{\top} & O
\end{array}\right)
$$

where $\boldsymbol{N}^{\top}$ is the transpose matrix of $\boldsymbol{N}$. The matrix $\boldsymbol{N}$ is called the biadjacency matrix of $\Gamma$. It is not hard to see that

$$
F_{2 i}(\boldsymbol{A})=\left(\begin{array}{cc}
\mathscr{F}_{0, i}\left(\boldsymbol{N} \boldsymbol{N}^{\top}\right) & \boldsymbol{O} \\
\boldsymbol{O} & \mathscr{F}_{0, i}\left(\boldsymbol{N}^{\top} \boldsymbol{N}\right)
\end{array}\right)
$$

Since each entry of $F_{2 i}(\boldsymbol{A})$ is non-negative [28], each entry of $\mathscr{F}_{0, i}\left(\boldsymbol{N} \boldsymbol{N}^{\top}\right)$ is also non-negative.

\section{LiNEAR PROGRAMMING BOUND FOR BIPARTITE REGULAR GRAPHS}

In this section, we give a linear programming bound for bipartite regular graphs. For general regular graphs, a linear programming bound was obtained by Nozaki [27].

THEOREM 3.1. Let $\Gamma$ be a connected bipartite $k$-regular graph with $v$ vertices. Let $\left\{ \pm \tau_{0}, \ldots, \pm \tau_{d}\right\}$ be the set of distinct eigenvalues of $\Gamma$, where $\tau_{0}=k$. If there exists a polynomial $f(x)=\sum_{i=0}^{t} f_{i} \mathscr{F}_{0, i}(x)$ such that $f\left(k^{2}\right)>0, f\left(\tau_{i}^{2}\right) \leqslant 0$ for each $i \in$ $\{1, \ldots, d\}, f_{0}>0$, and $f_{j} \geqslant 0$ for each $j \in\{1, \ldots, t\}$, then

$$
v \leqslant \frac{2 f\left(k^{2}\right)}{f_{0}} .
$$

Equality holds if and only if for each $i \in\{1, \ldots, d\}, f\left(\tau_{i}^{2}\right)=0$ and for each $j \in$ $\{1, \ldots, t\}, \operatorname{tr}\left(f_{j} \mathscr{F}_{0, j}\left(\boldsymbol{N} \boldsymbol{N}^{\top}\right)\right)=0$, and $\operatorname{tr}\left(f_{j} \mathscr{F}_{0, j}\left(\boldsymbol{N}^{\top} \boldsymbol{N}\right)\right)=0$, where $\boldsymbol{N}$ is the biadjacency matrix of $\Gamma$. If equality holds and $f_{j}>0$ for each $j \in\{1, \ldots, t\}$, then the girth of $\Gamma$ is at least $2 t+2$.

Proof. From the spectral decomposition $\boldsymbol{N} \boldsymbol{N}^{\top}=\sum_{i=0}^{d} \tau_{i}^{2} \boldsymbol{E}_{i}$, we deduce that

$$
f\left(k^{2}\right) \boldsymbol{E}_{0}+\sum_{i=1}^{d} f\left(\tau_{i}^{2}\right) \boldsymbol{E}_{i}=f\left(\boldsymbol{N} \boldsymbol{N}^{\top}\right)=\sum_{i=0}^{t} f_{i} \mathscr{F}_{0, i}\left(\boldsymbol{N} \boldsymbol{N}^{\top}\right)=f_{0} \boldsymbol{I}+\sum_{i=1}^{t} f_{i} \mathscr{F}_{0, i}\left(\boldsymbol{N} \boldsymbol{N}^{\top}\right),
$$

where $\boldsymbol{I}$ is the identity matrix, $\boldsymbol{E}_{0}=(2 / v) \boldsymbol{J}$, and $\boldsymbol{J}$ is the all-ones matrix. Taking traces in both sides of (11), we get that

$$
\begin{aligned}
& f\left(k^{2}\right)=\operatorname{tr}\left(f\left(k^{2}\right) \boldsymbol{E}_{0}\right) \geqslant \operatorname{tr}(\left.f\left(k^{2}\right) \boldsymbol{E}_{0}+\sum_{i=1}^{d} f\left(\tau_{i}^{2}\right) \boldsymbol{E}_{i}\right) \\
&=\operatorname{tr}\left(f_{0} \boldsymbol{I}+\sum_{j=1}^{t} f_{j} \mathscr{F}_{0, j}\left(\boldsymbol{N} \boldsymbol{N}^{\top}\right)\right) \geqslant \operatorname{tr}\left(f_{0} \boldsymbol{I}\right)=\frac{v f_{0}}{2} .
\end{aligned}
$$

Therefore, $v \leqslant 2 f\left(k^{2}\right) / f_{0}$. By using $\mathscr{F}_{0, j}\left(\boldsymbol{N}^{\top} \boldsymbol{N}\right)$, we can obtain the same bound as (10). 
If equality holds in (10), then for each $i \in\{1, \ldots, d\}, f\left(\tau_{i}^{2}\right)=0$ and for each $j \in\{1, \ldots, t\}, \operatorname{tr}\left(f_{j} \mathscr{F}_{0, j}\left(\boldsymbol{N} \boldsymbol{N}^{\top}\right)\right)=0$ and $\operatorname{tr}\left(f_{j} \mathscr{F}_{0, j}\left(\boldsymbol{N}^{\top} \boldsymbol{N}\right)\right)=0$. For the adjacency matrix $\boldsymbol{A}$, the $(u, v)$-entry of $F_{j}(\boldsymbol{A})$ is the number of non-backtracking walks of length $j$ from $u$ to $v$ [28]. Since (9) and $f_{j}>0$ for each $j \in\{1, \ldots, t\}$, there is no nonbacktracking walk of length $2 j$ from $u$ to $v$ for each $j \in\{1, \ldots, t\}$. Since $\Gamma$ is bipartite, the girth of $\Gamma$ is at least $2 t+2$.

\section{UPPER BOUND FOR BIPARTITE GRAPHS WITH GIVEN SECOND EIGENVALUE}

In this section, we obtain an upper bound on $b(k, \theta)$ using the bipartite linear programming bound given by Theorem 3.1. Let $c>0$ be a real number and $t \geqslant 4$ be an integer. Let $\boldsymbol{B}(k, t, c)$ be the $t \times t$ tridiagonal matrix with lower diagonal $(1, \ldots, 1, c, k)$, upper diagonal $(k, k-1, \ldots, k-1, k-c)$, and constant row sum $k$. Let

$$
\boldsymbol{B}(k, 3,1)=\left(\begin{array}{ccc}
0 & k & 0 \\
1 & 0 & k-1 \\
0 & k & 0
\end{array}\right) .
$$

THEOREM 4.1. If $\theta$ is the second largest eigenvalue of $\boldsymbol{B}(k, t, c)$, then

$$
b(k, \theta) \leqslant M(k, t, c)=2\left(\sum_{i=0}^{t-4}(k-1)^{i}+\frac{(k-1)^{t-3}}{c}+\frac{(k-1)^{t-2}}{c}\right) .
$$

Equality holds if and only if there exists a bipartite distance-regular graph whose quotient matrix with respect to the distance-partition from a vertex is $\boldsymbol{B}(k, t, c)$ for $1 \leqslant c<k$ or $\boldsymbol{B}(k, t-1,1)$ for $c=k$.

Proof. We first calculate the characteristic polynomial of $\boldsymbol{B}(k, t, c)$. The polynomials $F_{i}, G_{i}, \mathscr{F}_{i}$, and $\mathscr{G}_{i}$ are defined in Section 2. Note that $F_{i}(x)$ is the characteristic polynomial of the principal $i \times i$ matrix formed by the first $i$ rows and $i$ columns of $\boldsymbol{B}(k, t, 1)$ for $t>i+1$. By this fact and equations (4) and (6), we can compute

$$
\begin{aligned}
& |x \boldsymbol{I}-\boldsymbol{B}(k, t, c)| \\
& =\left|\begin{array}{cccccc}
x & -k & & & & \\
-1 & x & -(k-1) & & & \\
& \ddots & \ddots & \ddots & & \\
& & -1 & x & -(k-1) & \\
& & & -c & x & -(k-c) \\
& & & & -k & x
\end{array}\right| \\
& =k\left|\begin{array}{ccccc}
x & -k & & & \\
-1 & x & -(k-1) & \\
& \ddots & \ddots & \ddots & \\
& -1 & x & 0 \\
& & & -c-(k-c)
\end{array}\right|+x\left|\begin{array}{ccccc}
x & -k & & & \\
-1 & x & -(k-1) & \\
& \ddots & \ddots & \ddots & \\
& & -1 & x & -(k-1) \\
& & -c & x
\end{array}\right| \\
& =-k(k-c) F_{t-2}(x)+x\left(x F_{t-2}(x)-c(k-1) F_{t-3}(x)\right) \\
& =c\left(F_{t-2}(x)-(k-1)^{2} F_{t-4}(x)\right)+\left(x^{2}-k^{2}\right) F_{t-2}(x) \\
& =c\left(\left(x^{2}-k^{2}\right) F_{t-4}(x)+F_{t-4}(x)-(k-1)^{2} F_{t-6}(x)\right)+\left(x^{2}-k^{2}\right) F_{t-2}(x) \\
& =\left(x^{2}-k^{2}\right)\left(c \sum_{i=0}^{\lfloor(t-4) / 2\rfloor} F_{t-4-2 i}(x)+F_{t-2}(x)\right) \text {. }
\end{aligned}
$$


Note that

$$
c \sum_{i=0}^{\lfloor(t-4) / 2\rfloor} F_{t-4-2 i}(x)+F_{t-2}(x)=(c-1) G_{t-4}(x)+G_{t-2}(x) .
$$

Since the zeros of $\mathscr{G}_{\epsilon, s-1}$ and $\mathscr{G}_{\epsilon, s}$ interlace on $(0,4(k-1))$, each zero of $(c-1) \mathscr{G}_{\epsilon, s-1}+$ $\mathscr{G}_{\epsilon, s}$ is simple and belongs to $(0,4(k-1))$ except for the smallest zero. For $c=k$ the smallest zero is equal to 0 because $(k-1) \mathscr{G}_{\epsilon, s-1}(0)+\mathscr{G}_{\epsilon, s}(0)=0$ by $(5)$ and $(7)$. For $c>k$, the smallest zero is negative. From $x^{\epsilon}\left(x^{2}-k^{2}\right)\left((c-1) \mathscr{G}_{\epsilon, s-1}\left(x^{2}\right)+\mathscr{G}_{\epsilon, s}\left(x^{2}\right)\right)=$ $\left(x^{2}-k^{2}\right)\left((c-1) G_{2 s-2+\epsilon}(x)+G_{2 s+\epsilon}(x)\right)$, each non-zero real eigenvalue of $\boldsymbol{B}(k, t, c)$ has multiplicity 1 , and if $c>k$, then $\boldsymbol{B}(k, t, c)$ has imaginary eigenvalues.

Let $\mathfrak{f}_{1}(x)$ be the polynomial

$$
\mathfrak{f}_{1}(x)=\frac{\left((c-1) G_{t-4}(x)+G_{t-2}(x)\right)^{2}}{x^{2}-\theta^{2}}=\sum_{i=0}^{t-3} f_{i} \mathscr{F}_{0, i}\left(x^{2}\right) .
$$

We show that $\mathfrak{f}_{2}(x)=\sum_{i=0}^{t-3} f_{i} \mathscr{F}_{0, i}(x)$ satisfies the condition of the linear programming bound from Theorem 3.1 for bipartite graphs. Note that $\mathfrak{f}_{2}\left(k^{2}\right)=\mathfrak{f}_{1}(k)>0$, and $\mathfrak{f}_{2}\left(\lambda^{2}\right)=\mathfrak{f}_{1}(\lambda) \leqslant 0$ for each $\lambda \in[-\theta, \theta]$. It suffices to show that $f_{i}>0$ for each $i \in\{0,1, \ldots, t-3\}$.

The polynomial $\mathfrak{f}_{1}(x)$ can be expressed by

$$
\begin{aligned}
\mathfrak{f}_{1}(x) & =\frac{(c-1) G_{t-4}(x)+G_{t-2}(x)}{x^{2}-\theta^{2}}\left(c \sum_{i=0}^{\lfloor t / 2\rfloor-2} F_{t-4-2 i}(x)+F_{t-2}(x)\right) \\
& =x^{2 \epsilon} \frac{(c-1) \mathscr{G}_{\epsilon,\lfloor t / 2\rfloor-2}\left(x^{2}\right)+\mathscr{G}_{\epsilon,\lfloor t / 2\rfloor-1}\left(x^{2}\right)}{x^{2}-\theta^{2}}\left(c \sum_{i=0}^{\lfloor t / 2\rfloor-2} \mathscr{F}_{\epsilon, i}\left(x^{2}\right)+\mathscr{F}_{\epsilon,\lfloor t / 2\rfloor-1}\left(x^{2}\right)\right),
\end{aligned}
$$

where $\epsilon=0$ if $t$ is even, and $\epsilon=1$ if $t$ is odd. Thus,

$$
\mathfrak{f}_{2}(x)=x^{\epsilon} \frac{(c-1) \mathscr{G}_{\epsilon,\lfloor t / 2\rfloor-2}(x)+\mathscr{G}_{\epsilon,\lfloor t / 2\rfloor-1}(x)}{x-\theta^{2}}\left(c \sum_{i=0}^{\lfloor t / 2\rfloor-2} \mathscr{F}_{\epsilon, i}(x)+\mathscr{F}_{\epsilon,\lfloor t / 2\rfloor-1}(x)\right) \text {. }
$$

By Proposition 3.2 in [11], $g(x)=\left((c-1) \mathscr{G}_{\epsilon,\lfloor t / 2\rfloor-2}+\mathscr{G}_{\epsilon, \mid t / 2\rfloor-1}\right) /\left(x-\theta^{2}\right)$ has positive coefficients in terms of $\mathscr{G}_{\epsilon, 0}, \mathscr{G}_{\epsilon, 1}, \ldots, \mathscr{G}_{\epsilon, \mid t / 2\rfloor-2}$. This implies that $g(x)$ has positive coefficients in terms of $\mathscr{F}_{\epsilon, 0}, \mathscr{F}_{\epsilon, 1}, \ldots, \mathscr{F}_{\epsilon,\lfloor t / 2\rfloor-2}$. Therefore $f_{i}>0$ for each $i=\{0,1, \ldots, t-3\}$ by Lemma 2.1 .

The polynomial $g(x)$ can be expressed by $g(x)=\sum_{i=0}^{\lfloor t / 2\rfloor-2} g_{i} \mathscr{F}_{\epsilon, i}(x)$. By Lemma 2.1, we have

$$
f_{0}=\sum_{i=0}^{\lfloor t / 2\rfloor-2} c k^{\epsilon} g_{i} \mathscr{F}_{\epsilon, i}\left(k^{2}\right)=c k^{\epsilon} g\left(k^{2}\right) .
$$

By applying Theorem 3.1 to the polynomial $\mathfrak{f}_{2}(x)$, we have

$$
\begin{aligned}
b(k, \theta) & \leqslant \frac{2 \mathfrak{f}_{2}\left(k^{2}\right)}{f_{0}}=2 k^{\epsilon}\left(\sum_{i=0}^{\lfloor t / 2\rfloor-2} \mathscr{F}_{\epsilon, i}\left(k^{2}\right)+\mathscr{F}_{\epsilon,\lfloor t / 2\rfloor-1}\left(k^{2}\right) / c\right) \\
& =2\left(\sum_{i=0}^{t-4}(k-1)^{i}+\frac{(k-1)^{t-3}}{c}+\frac{(k-1)^{t-2}}{c}\right) .
\end{aligned}
$$

By Theorem 3.1, the bipartite graph attaining the bound $M(k, t, c)$ has girth at least $2 t-4$, and at most $t$ distinct eigenvalues. Since the diameter is at most $t-1$, the graph satisfies $g \geqslant 2 d-2$, where $g$ is the girth and $d$ is the diameter. By $g \geqslant 2 d-2$, the 
graph becomes a distance-regular graph [1, Theorem 4.4], [29], and it must have the quotient matrix $\boldsymbol{B}(k, t, c)$ for $1 \leqslant c<k$, or $\boldsymbol{B}(k, t-1,1)$ for $c=k$ (see Proposition 4.6 below). Conversely the distance-regular graph with the quotient matrix $\boldsymbol{B}(k, t, c)$ clearly attains the bound $M(k, t, c)$.

Note that $\Gamma$ is a distance-regular graph with the quotient matrix $\boldsymbol{B}(k, d+1, c)$ if and only if $\Gamma$ is a connected bipartite $k$-regular graph that has only $d+1$ distinct eigenvalues, and whose girth is at least $2 d-2$. Table 1 shows the known examples attaining the bound $M(k, d+1, c)$ [7, Section 6.11].

EXAMPLE 4.2. Recall that $v(k, \theta)$ denotes the maximum order of a connected (not necessarily bipartite) $k$-regular graph whose second largest eigenvalue is at most $\theta$. We have $v(3,1)=10$, which is attained by the Petersen graph $[10]$ and $b(3,1)=8$ from Table 1 , which is attained by the bipartite incidence graph of the symmetric $(4,3,2)$-design.

The following is the bipartite version of Theorem 5 in [27].

COROLlaRY 4.3. Let $\Gamma$ be a bipartite distance-regular graph of order $n$ with quotient matrix $\boldsymbol{B}(k, t, c)$ with respect to the distance-partition from a vertex. Then $\lambda_{2}(\Gamma) \leqslant$ $\lambda_{2}\left(\Gamma^{\prime}\right)$ for any bipartite $k$-regular graph $\Gamma^{\prime}$ of order $n$.

Proof. Assume that there exists a graph $\Gamma^{\prime}$ of order $n$ such that $\lambda_{2}\left(\Gamma^{\prime}\right)<\lambda_{2}(\Gamma)$. Then $\Gamma^{\prime}$ also attains the bound from Theorem 4.1. This implies that $\Gamma^{\prime}$ must have the eigenvalue $\lambda_{2}(\Gamma)$, which is a contradiction.

Let $\mu^{(j)}\left(\right.$ resp. $\left.\lambda^{(j)}\right)$ denote the largest zero of $F_{j}(x)\left(\right.$ resp. $\left.G_{j}(x)\right)$.

Proposition 4.4. For each $\theta \in[0,2 \sqrt{k-1})$, there exist $t, c$ such that $\theta$ is the second largest eigenvalue of $\boldsymbol{B}(k, t, c)$.

Proof. Note that $\lambda^{(j)}<\mu^{(j)}$ for $j \geqslant 1$ because $G_{j}(x)=\sum_{i=0}^{\lfloor j / 2\rfloor} F_{j-2 i}(x)>0$ for $x \geqslant \mu^{(j)}$. The second eigenvalue $\lambda_{2}(t, c)$ of $\boldsymbol{B}(k, t, c)$ is equal to the largest zero of $(c-1) G_{t-4}(x)+G_{t-2}(x)$. Since the zeros of $\mathscr{G}_{\epsilon,\lfloor t / 2\rfloor-2}$ and $\mathscr{G}_{\epsilon,\lfloor t / 2\rfloor-1}$ interlace, $\lambda_{2}(t, c)$ is a monotonically decreasing function in $c$. In particular, $\lim _{c \rightarrow \infty} \lambda_{2}(t, c)=\lambda^{(t-4)}$ with $t \geqslant 5, \lambda_{2}(t, 1)=\lambda^{(t-2)}$, and $\lim _{c \rightarrow 0} \lambda_{2}(t, c)=\mu^{(t-2)}$. The largest zero $r^{(j)}$ of $G_{j}(x)+G_{j-1}(x)$ can be expressed by $r^{(j)}=2 \sqrt{k-1} \cos \alpha$, where $\pi /(j+1)<\alpha<$ $\pi / j$ [6, Section III.3]. For $\lambda^{(j)}=2 \sqrt{k-1} \cos \beta$, it follows from $r^{(j)}<\lambda^{(j)}$ that $\beta<$ $\alpha<\pi / j$. This implies that the possible value $\lambda_{2}(t, c)$ is between $\lim _{c \rightarrow k} \lambda_{2}(4, c)=0$ and $\lim _{t \rightarrow \infty} \lambda_{2}(t, 1)=2 \sqrt{k-1}$. Therefore for each $\theta \in[0,2 \sqrt{k-1})$, there exist $t, c$ such that $\lambda$ is the second eigenvalue of $\boldsymbol{B}(k, t, c)$.

Note that for $\theta \in\left(\lambda^{(t-2)}, \mu^{(t-2)}\right], \theta$ is the second eigenvalue of both $\boldsymbol{B}\left(k, t, c_{1}\right)$ and $\boldsymbol{B}\left(k, t+2, c_{2}\right)$ for some $c_{1}, c_{2}$ with $0 \leqslant c_{1}<1, c_{2}>0$. By the following proposition, we may assume $c \geqslant 1$ in Theorem 4.1 to obtain better bounds.

Proposition 4.5. Let $\theta \in\left(\lambda^{(t-2)}, \mu^{(t-2)}\right]$. Suppose $c_{1}$ and $c_{2}$ satisfy that $0 \leqslant c_{1}<1$, $c_{2}>0$ and the second largest eigenvalues of $\boldsymbol{B}\left(k, t, c_{1}\right)$ and $\boldsymbol{B}\left(k, t+2, c_{2}\right)$ are $\theta$. Then we have $M\left(k, t, c_{1}\right)>M\left(k, t+2, c_{2}\right)$.

Proof. Since $\left(c_{1}-1\right) G_{t-4}(\theta)+G_{t-2}(\theta)=0$ holds, we have

$$
c_{1}=-\frac{G_{t-2}(\theta)-G_{t-4}(\theta)}{G_{t-4}(\theta)}=-\frac{F_{t-2}(\theta)}{G_{t-4}(\theta)} .
$$


TABLE 1 . Known bipartite graphs meeting the bound $M(k, d+1, c)$

\begin{tabular}{|c|c|c|c|c|c|}
\hline$k$ & $\theta$ & $b(k, \theta)$ & $d$ & $c$ & Name \\
\hline 2 & $2 \cos (2 \pi / n)$ & $n($ even $)$ & $n / 2$ & 1 & $n$-cycle $C_{n}$ \\
$k$ & 0 & $2 k$ & 2 & 1 & Complete bipartite graph $K_{k, k}$ \\
$k$ & $\sqrt{k-\tau}$ & $2(1+k(k-1) / \tau)$ & 3 & $\tau$ & Symmetric $(v, k, \tau)$-design \\
$r^{2}-r+1$ & $r$ & $2\left(r^{2}+1\right) \times\left(r^{2}-r+1\right)$ & 4 & $(r-1)^{2}$ & $p g\left(r^{2}-r+1, r^{2}-r+1,(r-1)^{2}\right)$ \\
$q$ & $\sqrt{q}$ & $2 q^{2}$ & 4 & $q-1$ & $A G(2, q)$ minus a parallel class \\
$q+1$ & $\sqrt{2 q}$ & $2 \sum_{i=0}^{3} q^{i}$ & 4 & 1 & $G Q(q, q)$ \\
$q+1$ & $\sqrt{3 q}$ & $2 \sum_{i=0}^{5} q^{i}$ & 6 & 1 & $G H(q, q)$ \\
6 & 2 & 162 & 4 & 2 & $p g(6,6,2)$ \\
\hline
\end{tabular}

$A G(2, q)$ : affine plane, $G Q(q, q)$ : generalized quadrangle, $G H(q, q)$ : generalized hexagon, $p g$ : partial geometry, $q$ : prime power, $r$ : power of 2 ,

We use the bipartite incidence graph of an incidence structure. 
Similarly $c_{2}=-F_{t}(\theta) / G_{t-2}(\theta)$ holds. By $\theta>\lambda^{(t-2)}$, we have $F_{t-2}(\theta)=-c_{1} G_{t-4}(\theta)<$ 0 and $F_{t}(\theta)=-c_{2} G_{t-2}(\theta)<0$. It therefore follows that

$$
\begin{aligned}
M\left(k, t, c_{1}\right)-M\left(k, t+2, c_{2}\right) & =2 k(k-1)^{t-2}\left(\frac{1}{c_{1}}-1-\frac{1}{c_{2}}(k-1)^{2}\right) \\
& =2 k(k-1)^{t-2}\left(-\frac{G_{t-4}(\theta)}{F_{t-2}(\theta)}-1+(k-1)^{2} \frac{G_{t-2}(\theta)}{F_{t}(\theta)}\right) \\
& =2 k(k-1)^{t-2}\left(-\frac{G_{t-2}(\theta)}{F_{t-2}(\theta)}+(k-1)^{2} \frac{G_{t-2}(\theta)}{F_{t}(\theta)}\right) \\
& =\frac{2 k(k-1)^{t-2} G_{t-2}(\theta)}{F_{t-2}(\theta) F_{t}(\theta)}\left(-F_{t}(\theta)+(k-1)^{2} F_{t-2}(\theta)\right) \\
& =\frac{2 k(k-1)^{t-2}\left(k^{2}-\theta^{2}\right) G_{t-2}(\theta)^{2}}{F_{t-2}(\theta) F_{t}(\theta)}>0 .
\end{aligned}
$$

For $\theta \in\left(\lambda^{(t-4)}, \lambda^{(t-3)}\right], \theta$ is the second eigenvalue of both $\boldsymbol{B}\left(k, t, c_{1}\right)$ and $\boldsymbol{B}(k, t-$ $1, c_{2}$ ) for some $c_{1}, c_{2}$ with $c_{1} \geqslant 1, c_{2} \geqslant 1$. It follows that

$$
\begin{aligned}
\frac{1}{c_{1}} & =-\frac{G_{t-4}(\theta)}{F_{t-2}(\theta)}=-\frac{G_{t-2}(\theta)}{F_{t-2}(\theta)}+1=-\frac{\lambda G_{t-3}(\theta)-(k-1) G_{t-4}(\theta)}{F_{t-2}(\theta)}+1 \\
& =-\frac{\lambda G_{t-3}(\theta)}{F_{t-2}(\theta)}-\frac{k-1}{c_{1}}+1,
\end{aligned}
$$

and hence

$$
\frac{k}{c_{1}}=-\frac{\lambda G_{t-3}(\theta)}{F_{t-2}(\theta)}+1
$$

for $t \geqslant 4$. Thus, if $\theta=\lambda^{(t-3)}$, then $c_{1}=k$. This implies that $k \leqslant c_{1}$. By the following proposition, we may assume $1 \leqslant c<k$ in Theorem 4.1 to obtain better bounds.

Proposition 4.6. Let $\theta \in\left(\lambda^{(t-4)}, \lambda^{(t-3)}\right]$. Suppose $c_{1}$ and $c_{2}$ satisfy that $k \leqslant c_{1}$, $c_{2} \geqslant 1$ and the second largest eigenvalues of $\boldsymbol{B}\left(k, t, c_{1}\right)$ and $\boldsymbol{B}\left(k, t-1, c_{2}\right)$ are $\theta$. Then we have $M\left(k, t, c_{1}\right) \geqslant M\left(k, t-1, c_{2}\right)$. Moreover, equality holds if and only if $c_{1}=k$ and $c_{2}=1$.

Proof. From $c_{2}=-F_{t-3}(\theta) / G_{t-5}(\theta)$ and (12), we have

$$
\begin{aligned}
\frac{M\left(k, t, c_{1}\right)-M\left(k, t-1, c_{2}\right)}{2(k-1)^{t-4}} & =1+\frac{k-1}{c_{1}}+\frac{(k-1)^{2}}{c_{1}}-\frac{1}{c_{2}}-\frac{k-1}{c_{2}} \\
& =1-\frac{1}{c_{2}}+(k-1)\left(\frac{k}{c_{1}}-\frac{1}{c_{2}}\right) \\
& =1+\frac{G_{t-5}(\theta)}{F_{t-3}(\theta)}+(k-1)\left(-\frac{x G_{t-3}(\theta)}{F_{t-2}(\theta)}+1+\frac{G_{t-5}(\theta)}{F_{t-3}(\theta)}\right) \\
& =\frac{G_{t-3}(\theta)}{F_{t-3}(\theta)}-(k-1)^{2} \frac{F_{t-4}(\theta) G_{t-3}(\theta)}{F_{t-2}(\theta) F_{t-3}(\theta)} \\
& =\frac{G_{t-3}(\theta)}{F_{t-3}(\theta) F_{t-2}(\theta)}\left(F_{t-2}(\theta)-(k-1)^{2} F_{t-4}(\theta)\right) \\
& =\left(x^{2}-k^{2}\right) \frac{G_{t-3}(\theta) G_{t-4}(\theta)}{F_{t-3}(\theta) F_{t-2}(\theta)} \geqslant 0 .
\end{aligned}
$$

This implies the proposition.

The above results imply the following theorem. 
THEOREM 4.7. Let $\lambda^{(j)}$ be the largest zero of $G_{j}(x)$ for $j \geqslant 1$. Then

$$
\bigcup_{j=1}^{\infty}\left(\lambda^{(j)}, \lambda^{(j+1)}\right]=(0,2 \sqrt{k-1})
$$

If $t \geqslant 4$ satisfies $\lambda^{(t-3)}<\theta \leqslant \lambda^{(t-2)}$, then

$$
b(k, \theta) \leqslant M(k, t, c)=2\left(\sum_{i=0}^{t-4}(k-1)^{i}+\frac{(k-1)^{t-3}}{c}+\frac{(k-1)^{t-2}}{c}\right)
$$

where $c=-F_{t-2}(\theta) / G_{t-4}(\theta)$.

The following results in $[18,19]$ are obtained as corollaries of Theorem 4.7.

COROLlaRY 4.8 ([18]). Let $\Gamma$ be a bipartite $n$-regular graph with $2 m$ nodes. If $\lambda_{2}(\Gamma) \leqslant$ $\sqrt{n-1}$, then

or, equivalently,

$$
m \leqslant 1+\frac{n(n-1)}{n-\lambda_{2}^{2}(\Gamma)}
$$

$$
\lambda_{2}^{2}(\Gamma) \geqslant \sqrt{\frac{m n-n^{2}}{m-1}} .
$$

Proof. This is immediate by Theorem 4.7 for $t=4$. Indeed, since $\lambda^{(1)}$ is the largest zero of $G_{1}(x)=x$, we have $\lambda^{(1)}=0$. Since $\lambda^{(2)}$ is the largest zero of $G_{2}(x)=$ $x^{2}-(n-1)$, we have $\lambda^{(2)}=\sqrt{n-1}$. Since $c=-F_{2}(\theta) / G_{0}(\theta)=n-\theta^{2}$, we have $M(n, 4, c) / 2=1+n(n-1) /\left(n-\theta^{2}\right)$.

COROLlary 4.9 ([19, Theorem 4]). Let $\Gamma$ be a bipartite $n$-regular graph with $2 m$ nodes. If $\sqrt{n-1} \leqslant \lambda_{2}(\Gamma) \leqslant \sqrt{2(n-1)}$, then

$$
m \leqslant n+\frac{n(n-1)}{2 n-\lambda_{2}^{2}(\Gamma)-1} .
$$

Proof. This is immediate by Theorem 4.7 for $t=5$. Indeed, since $\lambda^{(2)}$ is the largest zero of $G_{2}(x)=x^{2}-(n-1)$, we have $\lambda^{(2)}=\sqrt{n-1}$. Since $\lambda^{(3)}$ is the largest zero of $G_{3}(x)=x\left(x^{2}-2(n-1)\right)$, we have $\lambda^{(3)}=\sqrt{2(n-1)}$. Since $c=-F_{3}(\theta) / G_{1}(\theta)=$ $2 n-\theta^{2}-1$, we have

$$
M(n, 5, c) / 2=n+n(n-1) /\left(2 n-\theta^{2}-1\right) .
$$

For $0<\theta \leqslant \sqrt{k-1}$, the inequality $b(k, \theta) \leqslant 2\left(\theta^{4}+\theta^{2}+1\right)$ was obtained by Teranishi and Yasuno [29, Proposition 7.1]. This bound is improved as follows.

Corollary 4.10. If $k^{1 / 4}<\theta \leqslant \sqrt{k-1}$, then

$$
b(k, \theta) \leqslant 2\left(1+\frac{k-1}{k-\theta^{2}}+\frac{(k-1)^{2}}{k-\theta^{2}}\right)<2\left(\theta^{4}+\theta^{2}+1\right) .
$$

Proof. Note that we have $\lambda^{(1)}=0, \lambda^{(2)}=\sqrt{k-1}$, and $c=-F_{2}(\theta) / G_{0}(\theta)=k-\theta^{2}$. By Theorem 4.7, for $0<\theta \leqslant \sqrt{k-1}$, we have

$$
b(k, \theta) \leqslant 2\left(1+(k-1) /\left(k-\theta^{2}\right)+(k-1)^{2} /\left(k-\theta^{2}\right)\right) .
$$

The inequality $1+\frac{k-1}{k-\theta^{2}}+\frac{(k-1)^{2}}{k-\theta^{2}}<\theta^{4}+\theta^{2}+1$ holds if and only if $k^{1 / 4}<\theta \leqslant \sqrt{k-1}$. The assertion therefore follows.

By Theorem 4.1, the following is immediate. 
COROLlARY 4.11. Let $\Gamma$ be a connected bipartite $k$-regular graph of order $v$. If $\theta$ is the second largest eigenvalue of $\boldsymbol{B}(k, t, c)$ and $v \geqslant M(k, t, c)$, then $\lambda_{2}(\Gamma) \geqslant \theta$ holds.

Li and Solé $[24$, Theorems 3 and 5$]$ showed that if $\Gamma$ is of girth $g=2 l$, then $\lambda_{2}(\Gamma) \geqslant$ $2 \cos (\pi / l)$. Corollary 4.11 improves this result because we have $v \geqslant M(k, l+1,1)$ when $g=2 l$ and $\theta=2 \cos (\pi / l)$ for $\boldsymbol{B}(k, l+1,1)$.

We prove that the bound (2) is better than the bound (1) for any $k$ and $\theta$. For (1) we have a similar theorem to Theorem 4.7. For $j \geqslant 0$, denote $\mathcal{G}_{j}(x)=\sum_{i=0}^{j} F_{i}(x)$.

THEOREM 4.12 ([10]). Let $r^{(j)}$ be the largest zero of $\mathcal{G}_{j}(x)$ for $j \geqslant 1$. Then $\bigcup_{j=1}^{\infty}\left(r^{(j)}, r^{(j+1)}\right]=(-1,2 \sqrt{k-1})$. If $t \geqslant 3$ satisfies $r^{(t-2)}<\theta \leqslant r^{(t-1)}$, then

$$
v(k, \theta) \leqslant N(k, t, c)=1+\sum_{i=0}^{t-3} k(k-1)^{i}+\frac{k(k-1)^{t-2}}{c},
$$

where $c=-F_{t-1}(\theta) / \mathcal{G}_{t-2}(\theta)$.

THEOREM 4.13. Let $k \geqslant 2$ be an integer. For $\theta \in(0,2 \sqrt{k-1})$, let $M\left(k, t_{1}, c_{1}\right)$ and $N\left(k, t_{2}, c_{2}\right)$ be defined as in Theorems 4.7 and 4.12 , where $c_{1}=-F_{t_{1}-2}(\theta) / G_{t_{1}-4}(\theta)$, $c_{2}=-F_{t_{2}-1}(\theta) / \mathcal{G}_{t_{2}-2}(\theta), \lambda^{\left(t_{1}-3\right)}<\theta \leqslant \lambda^{\left(t_{1}-2\right)}$, and $r^{\left(t_{2}-2\right)}<\theta \leqslant r^{\left(t_{2}-1\right)}$. Then

$$
M\left(k, t_{1}, c_{1}\right) \leqslant N\left(k, t_{2}, c_{2}\right) .
$$

Equality holds only if $t_{1}=t_{2}=t+1, \theta=\lambda^{(t-1)}, c_{1}=1$, and $c_{2}=k$.

Proof. Note that $\lambda^{(t-2)}<r^{(t-1)}<\lambda^{(t-1)}$ because $\mathcal{G}_{t-1}(x)=G_{t-1}(x)+G_{t-2}(x)$ for any $t \geqslant 3$.

Because $\theta \in(0,2 \sqrt{k-1})=\cup_{j \geqslant 3}\left(\lambda^{(j-2)}, \lambda^{(j-1)}\right]$, there is $t \geqslant 3$ such that $\theta \in$ $\left(\lambda^{(t-2)}, r^{(t-1)}\right] \cup\left(r^{(t-1)}, \lambda^{(t-1)}\right]$. We consider each of the two possible cases $\lambda^{(t-2)}<$ $\theta \leqslant r^{(t-1)}$ and $r^{(t-1)}<\theta \leqslant \lambda^{(t-1)}$ separately.

Suppose $\lambda^{(t-2)}<\theta \leqslant r^{(t-1)}$. Then $t_{1}=t+1$ and $t_{2}=t$. From Theorem 4.12, a simple calculation yields that

$$
N\left(k, t_{2}, c_{2}\right)=2 \sum_{i=0}^{t_{2}-3}(k-1)^{i}+(k-1)^{t_{2}-2}+\frac{k(k-1)^{t_{2}-2}}{c_{2}},
$$

and therefore,

$$
\begin{aligned}
N\left(k, t, c_{2}\right)-M\left(k, t+1, c_{1}\right) & =\left(1+\frac{k}{c_{2}}-\frac{2 k}{c_{1}}\right)(k-1)^{t-2} \\
& =\left(1-\frac{k \mathcal{G}_{t-2}(\theta)}{F_{t-1}(\theta)}+\frac{2 k G_{t-3}(\theta)}{F_{t-1}(\theta)}\right)(k-1)^{t-2} \\
& =\left(1-\frac{k G_{t-2}(\theta)-k G_{t-3}(\theta)}{G_{t-1}(\theta)-G_{t-3}(\theta)}\right)(k-1)^{t-2}
\end{aligned}
$$

Because the zeroes of $G_{t-2}$ and $G_{t-1}$ interlace, we get that $G_{t-1}(\theta)<0<G_{t-3}(\theta)$. Thus, $G_{t-1}(\theta)-G_{t-3}(\theta)<0$. If $G_{t-2}(\theta)-G_{t-3}(\theta)>0$, then it is clear that $N\left(k, t, c_{2}\right)>M\left(k, t+1, c_{1}\right)$. Otherwise, if $G_{t-2}(\theta)-G_{t-3}(\theta)<0$, then

$$
\begin{aligned}
\left|k G_{t-2}(\theta)-k G_{t-3}(\theta)\right|-\left|G_{t-1}(\theta)-G_{t-3}(\theta)\right| & =(k-1) G_{t-3}(\theta)+G_{t-1}(\theta)-k G_{t-2}(\theta) \\
& =(\theta-k) G_{t-2}(\theta)<0
\end{aligned}
$$

which implies that $N\left(k, t_{2}, c_{2}\right)>M\left(k, t_{1}, c_{1}\right)$. 
Suppose $r^{(t-1)}<\theta \leqslant \lambda^{(t-1)}$. Then $t_{1}=t+1$ and $t_{2}=t+1$. Thus we have

$$
\begin{aligned}
& N\left(k, t+1, c_{2}\right)-M\left(k, t+1, c_{1}\right)=\left(k+1+\frac{k(k-1)}{c_{2}}-\frac{2 k}{c_{1}}\right)(k-1)^{t-2} \\
& =\left(k+1-\frac{k(k-1) \mathcal{G}_{t-1}(\theta)}{F_{t}(\theta)}+\frac{2 k G_{t-3}(\theta)}{F_{t-1}(\theta)}\right)(k-1)^{t-2} \\
& =\left(k+1-\frac{k(k-1)\left(G_{t-1}(\theta)+G_{t-2}(\theta)\right)}{F_{t}(\theta)}+\frac{2 k\left(G_{t-1}(\theta)-F_{t-1}(\theta)\right)}{F_{t-1}(\theta)}\right)(k-1)^{t-2} \\
& =\left(-\frac{k(k-1) G_{t-1}(\theta)}{F_{t}(\theta)}+\frac{2 k G_{t-1}(\theta)}{F_{t-1}(\theta)}-(k-1)\left(1+k \frac{G_{t-2}(\theta)}{F_{t}(\theta)}\right)\right)(k-1)^{t-2} \\
& =\left(-\frac{k(k-1) G_{t-1}(\theta)}{F_{t}(\theta)}+\frac{2 k G_{t-1}(\theta)}{F_{t-1}(\theta)}-\frac{(k-1) \theta G_{t-1}(\theta)}{F_{t}(\theta)}\right)(k-1)^{t-2} \\
& =\left(\frac{k F_{t}(\theta)-k(k-1) F_{t-1}(\theta)}{F_{t}(\theta) F_{t-1}(\theta)}+\frac{k F_{t}(\theta)-\theta(k-1) F_{t-1}(\theta)}{F_{t}(\theta) F_{t-1}(\theta)}\right)(k-1)^{t-2} G_{t-1}(\theta) \\
& =\left(\frac{k(\theta-k) \mathcal{G}_{t-1}(\theta)}{F_{t}(\theta) F_{t-1}(\theta)}+\frac{\left(\theta^{2}-k^{2}\right) G_{t-2}(\theta)}{F_{t}(\theta) F_{t-1}(\theta)}\right)(k-1)^{t-2} G_{t-1}(\theta) \\
& =\left(\frac{k \mathcal{G}_{t-1}(\theta)+(\theta+k) G_{t-2}(\theta)}{F_{t}(\theta) F_{t-1}(\theta)}\right)(k-1)^{t-2}(\theta-k) G_{t-1}(\theta) \geqslant 0 .
\end{aligned}
$$

Equality holds only if $G_{t-1}(\theta)=0$ meaning that $\theta=\lambda^{(t-1)}$. Since $c_{1}=\frac{-F_{t-1}(\theta)}{G_{t-3}(\theta)}=$ $\frac{G_{t-3}(\theta)-G_{t-1}(\theta)}{G_{t-3}(\theta)}=1-\frac{G_{t-1}(\theta)}{G_{t-3}(\theta)}$ and $c_{2}=\frac{-F_{t}(\theta)}{\mathcal{G}_{t-1}(\theta)}=\frac{G_{t-2}(\theta)-G_{t}(\theta)}{G_{t-1}(\theta)+G_{t-2}(\theta)}=\frac{k G_{t-2}(\theta)}{G_{t-2}(\theta)}$, this means that $c_{1}=1$, and $c_{2}=k$.

\section{Non-EXistence of Certain Distance-Regular GRAPhS}

In this section, we prove the non-existence of the graph that attains the bound in Theorem 4.1 for $t>15$ and $t=12$. Namely we prove the following.

THEOREM 5.1. Let $k$ and $c$ be two integers such that $k \geqslant 3$ and $1 \leqslant c \leqslant k-1$. If $d=11$ or $d \geqslant 15$, there is no distance-regular graph $\Gamma$ with the quotient matrix $\boldsymbol{B}(k, d+1, c)$.

We prove Theorem 5.1 by the manner given by Fuglister [14]. Let $x=(t+$ $1 / t) \sqrt{k-1}$. The polynomial $G_{i}(x)$ can be expressed by

$$
G_{i}(x)=\frac{\sqrt{(k-1)^{i}}}{t^{i}\left(t^{2}-1\right)}\left(t^{2 i+2}-1\right) .
$$

The characteristic polynomial of $\boldsymbol{B}(k, d+1, c)$ is $\left(x^{2}-k^{2}\right)\left((c-1) G_{d-3}(x)+G_{d-1}(x)\right)$, and we have the expression

$$
\begin{aligned}
\mathscr{S}_{d}(x) & =(c-1) G_{d-3}(x)+G_{d-1}(x) \\
& =\frac{\sqrt{(k-1)^{d-1}}}{t^{d-1}\left(t^{2}-1\right)}\left(t^{2 d}+\frac{c-1}{k-1} t^{2 d-2}-\frac{c-1}{k-1} t^{2}-1\right) .
\end{aligned}
$$

Let $\theta$ be an eigenvalue of $\Gamma$ that is not $\pm k$. Put $\theta=(\tau+1 / \tau) \sqrt{k-1}$ for some complex number $\tau$. Let $n$ be the order of $\Gamma$. The multiplicity $m_{\theta}$ is given by

$$
m_{\theta}=\frac{n c k(k-c)(k-1)^{d-2}}{\left(k^{2}-\theta^{2}\right) \mathscr{S}_{d}^{\prime}(\theta) f_{d-1}(\theta)},
$$

where $\mathscr{S}_{d}^{\prime}(x)$ is the derivative with respect to $x, f_{d-1}=(x-1+c) G_{d-2}+(x-k+$ c) $G_{d-3}-(k-1) G_{d-4}$ and $G_{-1}=0$. From $\mathscr{S}_{d}(\theta)=0$, we can obtain

$$
\tau^{2 d-2}=\frac{(c-1) \tau^{2}+k-1}{(k-1) \tau^{2}+c-1} .
$$


Then we may calculate that

$$
f_{d-1}(\theta)=-\frac{c(k-c) \sqrt{(k-1)^{d-2}}}{\tau^{d-2}\left((k-1) \tau^{2}+c-1\right)}
$$

and

$$
\mathscr{S}_{d}^{\prime}(\theta)=\frac{2 \sqrt{(k-1)^{d-4}}\left[(d-1)(k-1)(c-1)\left(\tau^{4}+1\right)+\left(d(k-1)^{2}+(d-2)(c-1)^{2}\right) \tau^{2}\right]}{\tau^{d-2}\left(\tau^{2}-1\right)^{2}\left((k-1) \tau^{2}+c-1\right)} .
$$

From these equations, the multiplicity $m_{\theta}$ can be expressed by

$$
\begin{aligned}
m_{\theta} & =\frac{n k(k-1)\left(\tau^{2}-1\right)^{2}\left((c-1) \tau^{2}+k-1\right)\left((k-1) \tau^{2}+c-1\right)}{2\left(\theta^{2}-k^{2}\right) \tau^{2}\left[(d-1)(k-1)(c-1)\left(\tau^{4}+1\right)+\left(d(k-1)^{2}+(d-2)(c-1)^{2}\right) \tau^{2}\right]} \\
& =\frac{n k\left(\theta^{2}-4(k-1)\right)\left((c-1) \theta^{2}+(k-c)^{2}\right)}{2\left(\theta^{2}-k^{2}\right)\left[(d-1)(c-1) \theta^{2}+d(k-c)^{2}+2(c-1)(k-c)\right]}
\end{aligned}
$$

$$
=\frac{n k(k-1)(\phi-4)\left((c-1)(k-1) \phi+(k-c)^{2}\right)}{2\left((k-1) \phi-k^{2}\right)\left[(d-1)(c-1)(k-1) \phi+d(k-c)^{2}+2(c-1)(k-c)\right]},
$$

where $\theta^{2}=(k-1) \phi$. Unless $(k, c)=(2,1)$, expression (13) gives a non-trivial rational quadratic polynomial in $\phi$.

Set

$$
H_{d}(x)=\frac{\mathscr{S}_{d}(x)}{x^{\epsilon} \sqrt{(k-1)^{d-3-\epsilon}}}
$$

where $\epsilon=1$ if $d$ is even, and $\epsilon=0$ if $d$ is odd. Let $z=x^{2} /(k-1)$. For $u=t^{2}$, we have $z=(t+1 / t)^{2}=u+1 / u+2$. We compute

$$
H_{2 m+1-\epsilon}(z)=(c-1) P_{m-1, \epsilon}(z)+(k-1) P_{m, \epsilon}(z),
$$

where

$$
P_{i, \epsilon}(z)=\frac{u^{2 i+1-\epsilon}-1}{u^{i-\epsilon}(u+1)^{\epsilon}(u-1)} .
$$

Note that $P_{i, \epsilon}(z)$ satisfy the recurrence relation

$$
P_{i, \epsilon}(z)=(z-2) P_{i-1, \epsilon}(z)-P_{i-2, \epsilon}(z)
$$

with the initial conditions $P_{0, \epsilon}(z)=1-\epsilon, P_{1,1}(z)=1$ and $P_{1,0}(z)=z-1$. This implies that $P_{i, \epsilon}(z)$ is a monic polynomial of degree $i$ with integer coefficients. Table 2 shows some useful identities involving polynomials $P_{i, \epsilon}(z)$. By $(13)$, the polynomial $H_{d}(z)$ must split over the rationals into factors of degree at most 2 .

TABLE 2. Identities involving $P_{i, \epsilon}(z)$

\begin{tabular}{|l|}
\hline$d=2 m+1-\epsilon$ \\
\hline$P_{m, \epsilon}(z)=\left(u^{d}-1\right) / u^{m-\epsilon}(u-1)(u+1)^{\epsilon}$ \\
$P_{m-1, \epsilon}(z)=\left(u^{d-2}-1\right) / u^{m-1-\epsilon}(u-1)(u+1)^{\epsilon}$ \\
$P_{m-1, \epsilon}(z)+P_{m, \epsilon}(z)=(u+1)^{1-\epsilon}\left(u^{d-1}-1\right) / u^{m-\epsilon}(u-1)$ \\
$-P_{m-1, \epsilon}(z)+P_{m, \epsilon}(z)=\left(u^{d-1}+1\right) / u^{m-\epsilon}(u+1)^{\epsilon}$ \\
\hline
\end{tabular}


5.1. Case Analysis modulo 2. Let $c^{\prime}=c-1$ and $k^{\prime}=k-1$. If $c^{\prime}$ and $k^{\prime}$ have a factor in common, we may still factor out the content of $H_{d}(z)$. Call the resulting polynomial $\hat{H}_{d}(z)$. For $\hat{H}_{d}(z)$ modulo 2 , there are three cases A-C, which are listed in Table 3. For natural numbers $n$ and $a$, let $\operatorname{ord}_{n}(a)$ be the non-negative integer $s$ such that $a=n^{s} b$ and $b$ is an integer that is not divisible by $n$. Suppose $\operatorname{ord}_{n}(0)=\infty$.

TABLE 3. $\hat{H}_{d}(z)$ modulo $2, w \in\{1,3,5\}$

\begin{tabular}{|l|l|l|l|}
\hline Cases & Conditions & $\hat{H}_{d}(z)(\bmod 2)$ & $d$ \\
\hline $\mathrm{A}$ & $\operatorname{ord}_{2}\left(c^{\prime}\right)>\operatorname{ord}_{2}\left(k^{\prime}\right)$ & $P_{m, \epsilon}(z)$ & $d=2^{r} w$ \\
$\mathrm{~B}$ & $\operatorname{ord}_{2}\left(c^{\prime}\right)<\operatorname{ord}_{2}\left(k^{\prime}\right)$ & $P_{m-1, \epsilon}(z)$ & $d-2=2^{r} w$ \\
$\mathrm{C}$ & $\operatorname{ord}_{2}\left(c^{\prime}\right)=\operatorname{ord}_{2}\left(k^{\prime}\right)$ & $P_{m-1, \epsilon}(z)+P_{m, \epsilon}(z)$ & $d-1=2^{r} w$ \\
\hline
\end{tabular}

Each root of $\hat{H}_{d}(z)$ is a root of one of the three irreducible polynomials of degree at most 2 over GF(2), which are listed in Table 4 . There are also listed the results of the substitution $z=u+1 / u+2$, as well as the multiplicative orders modulo 2 of the roots of the polynomials in $u$.

TABLE 4. Irreducible polynomials over $\mathrm{GF}(2)$

\begin{tabular}{|l|l|l|}
\hline$f(z)$ & $z=u+1 / u+2$ & order of $u$ \\
\hline$z$ & $(u+1)^{2} / u$ & 1 \\
$z+1$ & $\left(u^{2}+u+1\right) / u$ & 3 \\
$z^{2}+z+1$ & $\left(u^{4}+u^{3}+u^{2}+u+1\right) / u^{2}$ & 5 \\
\hline
\end{tabular}

If an expression $u^{i}-1$ occurs as a factor of $\hat{H}_{d}(z)$ modulo 2 , then we must have $i=2^{r} w$ for $w \in\{1,3,5\}$. From the identities in Table 2 , we can obtain the possible values for the diameter $d$ in Table 3 .

5.2. Case analysis modulo 3. For $\hat{H}_{d}(z)$ modulo 3 , there are three cases a-d, which are listed in Table 5. If $\operatorname{ord}_{3}\left(c^{\prime}\right)=\operatorname{ord}_{3}\left(k^{\prime}\right)=m$, then let $c^{\prime \prime}=c^{\prime} / 3^{m}$ and $k^{\prime \prime}=k^{\prime} / 3^{m}$.

TABLE $5 . \hat{H}_{d}(z)$ modulo $3, w \in\{1,2,4,5,8,10\}$

\begin{tabular}{|l|l|l|l|}
\hline Cases & Conditions & $\hat{H}_{d}(z)(\bmod 2)$ & $d$ \\
\hline a & $\operatorname{ord}_{3}\left(c^{\prime}\right)>\operatorname{ord}_{3}\left(k^{\prime}\right)$ & $\pm P_{m, \epsilon}(z)$ & $d=3^{r} w$ \\
b & $\operatorname{ord}_{3}\left(c^{\prime}\right)<\operatorname{ord}_{3}\left(k^{\prime}\right)$ & $\pm P_{m-1, \epsilon}(z)$ & $d-2=3^{r} w$ \\
c & $\operatorname{ord}_{3}\left(c^{\prime}\right)=\operatorname{ord}_{3}\left(k^{\prime}\right), c^{\prime \prime} \equiv k^{\prime \prime}(\bmod 3)$ & $\pm\left(P_{m-1, \epsilon}(z)+P_{m, \epsilon}(z)\right)$ & $d-1=3^{r} w$ \\
d & $\operatorname{ord}_{3}\left(c^{\prime}\right)=\operatorname{ord}_{3}\left(k^{\prime}\right), c^{\prime \prime} \equiv-k^{\prime \prime}(\bmod 3)$ & $\pm\left(P_{m-1, \epsilon}(z)-P_{m, \epsilon}(z)\right)$ & $2 d-2=3^{r} w$ \\
\hline
\end{tabular}

There are six irreducible polynomials of degree at most 2 over GF(3), which are listed in Table 6 . We can obtain the possible values for the diameter $d$ in Table 5 by a similar way to modulo 2 . Here $w \in\{1,2,4,5,8,10\}$. 
TABLE 6. Irreducible polynomials over GF(3)

\begin{tabular}{|l|l|l|}
\hline$f(z)$ & $z=u+1 / u+2$ & order of $u$ \\
\hline$z-1$ & $(u-1)^{2} / u$ & 1 \\
$z$ & $(u+1)^{2} / u$ & 2 \\
$z+1$ & $\left(u^{2}+1\right) / u$ & 4 \\
$z^{2}-z-1$ & $\left(u^{2}-u-1\right)\left(u^{2}+u+1\right) / u^{2}$ & 8 \\
$z^{2}+1$ & $\left(u^{4}+u^{3}+u^{2}+u+1\right) / u^{2}$ & 5 \\
$z^{2}+z-1$ & $\left(u^{4}-u^{3}+u^{2}-u+1\right) / u^{2}$ & 10 \\
\hline
\end{tabular}

5.3. A BOUND FOR THE DIAMETER. Using a method similar to the one of Fuglister [14], we can obtain the possible values of $d$ in all cases $\mathrm{A}-\mathrm{C}$ and a-d, which are listed in Table 7.

TABle 7. Possible values of $d$

\begin{tabular}{|c|c|c|}
\hline \multicolumn{2}{|l|}{ Case } & \multirow[b]{2}{*}{ Possible values of $d$} \\
\hline$(\bmod 2)$ & $(\bmod 3)$ & \\
\hline \multirow[t]{3}{*}{ A } & $\mathrm{a}$ & $3-6,8,10,12,24$ \\
\hline & $\mathrm{b}$ & $3-6,8,10,12,20,32$ \\
\hline & $\mathrm{c}, \mathrm{d}$ & $3-6,10,16$ \\
\hline \multirow[t]{3}{*}{ B } & a & $3-6,8,10,12,18,162$ \\
\hline & $\mathrm{b}$ & $3-8,10,12,14,26$ \\
\hline & $\mathrm{c}, \mathrm{d}$ & $3-7,10,82$ \\
\hline \multirow[t]{4}{*}{$\mathrm{C}$} & $\mathrm{a}$ & $3-6,9,81$ \\
\hline & $\mathrm{b}$ & $3-7,11,17$ \\
\hline & $\mathrm{c}$ & $3-7,9,11,13,25$ \\
\hline & $\mathrm{d}$ & $3-7,13$ \\
\hline
\end{tabular}

We eliminate several choices of $d$ from Table 7 in this subsection.

Proposition 5.2. There does not exist a distance-regular graph $\Gamma$ with the quotient matrix $\boldsymbol{B}(k, d+1, c)$ for $d=17,18,20,32,81,82,162$.

Proof. Using a computer, we can obtain the factorization of $c^{\prime} P_{m-1, \epsilon}(z)+k^{\prime} P_{m, \epsilon}(z)$ modulo $p$ into irreducible polynomials for given $d, k^{\prime}$ and $c^{\prime}$.

For $d=18,81,82,162$, we can find an irreducible polynomial of degree at least 3 as a factor of $c^{\prime} P_{m-1, \epsilon}(z)+k^{\prime} P_{m, \epsilon}(z)$ over $\mathrm{GF}(5)$ for each pair $\left(c^{\prime}, k^{\prime}\right) \in \mathrm{GF}(5) \times \mathrm{GF}(5)$.

For $d=20,32$, we can find an irreducible polynomial of degree at least 3 as a factor of $c^{\prime} P_{m-1, \epsilon}(z)+k^{\prime} P_{m, \epsilon}(z)$ over $\operatorname{GF}(7)$ for each pair $\left(c^{\prime}, k^{\prime}\right) \in \mathrm{GF}(7) \times \mathrm{GF}(7)$.

For $d=17$, we can find an irreducible polynomial of degree at least 3 as a factor of $c^{\prime} P_{m-1, \epsilon}(z)+k^{\prime} P_{m, \epsilon}(z)$ over $\mathrm{GF}(43)$ for each pair $\left(c^{\prime}, k^{\prime}\right) \in \mathrm{GF}(43) \times \mathrm{GF}(43)$.

Bannai and Ito [4] proved the unimodal property of the multiplicities of the eigenvalues of Moore polygons. After this work, they also proved the rationality of the eigenvalues of Moore polygons [5]. The rationality of the eigenvalues is essential for the proof of the non-existence of Moore polygons [12]. In our case, the unimodal property of the multiplicities for the positive eigenvalues is easy. 
LEMMA 5.3. Let $\Gamma$ be a distance-regular graph with the quotient matrix $\boldsymbol{B}(k, d+1, c)$. Let $d^{\prime}=\lfloor(d-1) / 2\rfloor$, which is the number of the positive non-trivial eigenvalues. Let $\theta_{1}, \ldots, \theta_{d^{\prime}}$ be the positive non-trivial eigenvalues of $\Gamma$ with $\theta_{1}>\cdots>\theta_{d^{\prime}}$. Let $m_{\theta_{i}}$ be the multiplicity of $\theta_{i}$. Then it follows that

$$
m_{\theta_{1}}<m_{\theta_{2}}<\cdots<m_{\theta_{i-1}}<m_{\theta_{i}} \geqslant m_{\theta_{i+1}}>\cdots>m_{\theta_{d^{\prime}}}
$$

for some $i \in\left\{1, \ldots, d^{\prime}\right\}$.

Proof. The multiplicity $m_{\theta}$ of the eigenvalue $\theta$ can be expressed by equation (13). The function $m_{\theta}$ has no pole for $0<\phi<4$ and $k \geqslant 3$. This implies the unimodal property of the multiplicities.

It is known that

$$
G_{i}(x)=(k-1)^{i / 2} U_{i}\left(\frac{x}{2 \sqrt{k-1}}\right),
$$

where $U_{i}$ is the Chebyshev polynomial of degree $i$, which is defined by $U_{i}(\cos \theta)=$ $\sin ((i+1) \theta) / \sin \theta$ (see [13]). Thus the zeros of $G_{i}(x)$ are $2 \sqrt{k-1} \cos u_{j}^{(i)}$ for $j=$ $1, \ldots, i$, where $u_{j}^{(i)}=j \pi /(i+1)$. Since the expression

$$
\mathscr{S}_{d}(x)=(c-1) G_{d-3}(x)+G_{d-1}(x)=x G_{d-2}(x)-(k-c) G_{d-3}(x),
$$

the positive zeros $\theta_{i}=2 \sqrt{k-1} \cos \alpha_{i}$ of $\mathscr{S}_{d}(x)$ with $0<\alpha_{1}<\cdots<\alpha_{d^{\prime}}<\pi / 2$ satisfy $u_{i}^{(d-1)}<\alpha_{i}<u_{i}^{(d-2)}$ for each $i \in\left\{1, \ldots, d^{\prime}\right\}$.

Let $a(\phi)$ and $b(\phi)$ be the functions defined by

$$
\begin{aligned}
a(\phi) & =\frac{\phi-4}{(k-1) \phi-k^{2}}, \\
b(\phi) & =\frac{(c-1)(k-1) \phi+(k-c)^{2}}{(d-1)(c-1)(k-1) \phi+d(k-c)^{2}+2(c-1)(k-c)} \\
& =\frac{X_{\phi}}{(d-1) X_{\phi}+Y},
\end{aligned}
$$

where $X_{\phi}=(c-1)(k-1) \phi+(k-c)^{2}$ and $Y=(k-c)(k+c-2)$. Note that $m_{\theta}=n k(k-1) a(\phi) b(\phi) / 2$. Let $\theta_{\alpha}^{2}=(k-1) \alpha$ and $\theta_{\beta}^{2}=(k-1) \beta$. It follows that $m_{\theta_{\beta}}>m_{\theta_{\alpha}}$ if and only if

$$
\frac{a(\beta)}{a(\alpha)} \cdot \frac{b(\beta)}{b(\alpha)}>1
$$

Let $\alpha=4 \cos ^{2} v$ and $\beta=4 \cos ^{2} w$. By direct calculation,

$$
\frac{a(\beta)}{a(\alpha)}=1+\frac{\cos 2 v-\cos 2 w}{1-\cos 2 v} \cdot \frac{(k-2)^{2}}{(k-2)^{2}+2(k-1)(1-\cos 2 w)}
$$

and

Let

$$
\frac{b(\beta)}{b(\alpha)}=1-\frac{Y\left(X_{\alpha}-X_{\beta}\right)}{(d-1) X_{\alpha} X_{\beta}+Y X_{\alpha}}
$$

and

$$
A=\frac{\cos 2 v-\cos 2 w}{1-\cos 2 v} \cdot \frac{(k-2)^{2}}{(k-2)^{2}+2(k-1)(1-\cos 2 w)}
$$

Note that

$$
B=\frac{Y\left(X_{\alpha}-X_{\beta}\right)}{(d-1) X_{\alpha} X_{\beta}+Y X_{\alpha}} .
$$

$$
\frac{a(\beta)}{a(\alpha)} \cdot \frac{b(\beta)}{b(\alpha)}=(1+A)(1-B)=1+B\left(A\left(\frac{1}{B}-1\right)-1\right) .
$$

If $A(1 / B-1)>1$ holds, then $a(\beta) b(\beta) /(a(\alpha) b(\alpha))>1$. 
LEMMA 5.4. If $\pi / 4<v<w<\pi / 2$ holds, then it follows that

$$
A\left(\frac{1}{B}-1\right)>L(v, w)
$$

where $L(v, w):=\frac{3 \sqrt{3}(d-1)}{4}\left(1-\frac{2}{k}\right)^{2}(1+\cos 2 v) \sin ^{2} 2 w$.

Proof. We can calculate

$$
\frac{1}{B}-1=\frac{(d-1) X_{\alpha} X_{\beta}+Y X_{\beta}}{Y\left(X_{\alpha}-X_{\beta}\right)} .
$$

Since it follows that

$$
X_{\alpha}=((c-1)+(k-1) \cos 2 v)^{2}+(k-1)^{2}\left(1-\cos ^{2} 2 v\right) \geqslant(k-1)^{2} \sin ^{2} 2 v,
$$

we have

$$
(d-1) X_{\alpha} X_{\beta} \geqslant(d-1)(k-1)^{4} \sin ^{2} 2 v \sin ^{2} 2 w
$$

and

$$
Y X_{\beta} \geqslant(k-c)(k+c-2)(k-1)^{2} \sin ^{2} 2 w .
$$

It follows that

$$
Y\left(X_{\alpha}-X_{\beta}\right)=2(k-c)(k+c-2)(c-1)(k-1)(\cos 2 v-\cos 2 w) .
$$

For $1 \leqslant c \leqslant k-1$, the function $f(c)=(k-c)(k+c-2)(c-1)$ is maximum at $c=1+(k-1) / \sqrt{3}$. It therefore follows that

$$
Y\left(X_{\alpha}-X_{\beta}\right)<\frac{4}{3 \sqrt{3}}(k-1)^{4}(\cos 2 v-\cos 2 w) .
$$

Thus we obtain

$$
\begin{aligned}
\frac{1}{B}-1 & >\frac{3 \sqrt{3}\left((d-1)(k-1)^{2} \sin ^{2} 2 v \sin ^{2} 2 w+(k-c)(k+c-2) \sin ^{2} 2 w\right)}{4(k-1)^{2}(\cos 2 v-\cos 2 w)} \\
& >\frac{3 \sqrt{3}(d-1) \sin ^{2} 2 v \sin ^{2} 2 w}{4(\cos 2 v-\cos 2 w)}
\end{aligned}
$$

and hence

$$
\begin{aligned}
& A\left(\frac{1}{B}-1\right) \\
& >\frac{\cos 2 v-\cos 2 w}{1-\cos 2 v} \cdot \frac{(k-2)^{2}}{(k-2)^{2}+2(k-1)(1-\cos 2 w)} \cdot \frac{3 \sqrt{3}(d-1) \sin ^{2} 2 v \sin ^{2} 2 w}{4(\cos 2 v-\cos 2 w)} \\
& \quad>\frac{3 \sqrt{3}(d-1)}{4} \cdot \frac{(k-2)^{2}}{(k-2)^{2}+4(k-1)} \cdot(1+\cos 2 v) \sin ^{2} 2 w \\
& =\frac{3 \sqrt{3}(d-1)}{4}\left(1-\frac{2}{k}\right)^{2}(1+\cos 2 v) \sin ^{2} 2 w .
\end{aligned}
$$

LEMmA 5.5. Suppose $\alpha=4 \cos ^{2} v=\theta_{\alpha}^{2} /(k-1), \beta=4 \cos ^{2} w=\theta_{\beta}^{2} /(k-1)$, and $\pi / 4<v<w<\pi / 2$. If $L(v, w) \geqslant 1$, then $m_{\theta_{\alpha}}<m_{\theta_{\beta}}$.

Proof. By Lemma 5.4 and $B>0$, we have

$$
\begin{aligned}
\frac{m_{\theta_{\beta}}}{m_{\theta_{\alpha}}} & =\frac{a(\beta)}{a(\alpha)} \cdot \frac{b(\beta)}{b(\alpha)}=(1+A)(1-B) \\
& =1+B\left(A\left(\frac{1}{B}-1\right)-1\right)>1+B(L(v, w)-1) \geqslant 1 .
\end{aligned}
$$


Lemma 5.6. Let $\Gamma$ be a distance-regular graph with the quotient matrix $\boldsymbol{B}(k, d+1, c)$. Let $u_{j}^{(i)}=j \pi /(i+1)$ and $d^{\prime}=\lfloor(d-1) / 2\rfloor$. If $L\left(u_{d^{\prime}-j-1}^{(d-2)}, u_{d^{\prime}-j}^{(d-2)}\right) \geqslant 1$ for some integer $j$ with $0 \leqslant j<d^{\prime}-(d+3) / 4$, then the number of positive eigenvalues $\theta$ of $\Gamma$ such that $\theta^{2}$ is irrational is less than or equal to $j$.

Proof. The inequality $j<d^{\prime}-(d+3) / 4$ implies $u_{d^{\prime}-j-1}^{(d-2)}>\pi / 4$. If $\theta=2 \sqrt{k-1} \cos u$, we write $m_{\theta}=m(u)$. By Lemma 5.5 , we have $m\left(u_{d^{\prime}-j-1}^{(d-2)}\right)<m\left(u_{d^{\prime}-j}^{(d-2)}\right)$. Note that the eigenvalues $\theta_{i}=2 \sqrt{k-1} \cos \alpha_{i}$ satisfy $u_{i}^{(d-1)}<\alpha_{i}<u_{i}^{(d-2)}$. By $m\left(u_{d^{\prime}-j-1}^{(d-2)}\right)<$ $m\left(u_{d^{\prime}-j}^{(d-2)}\right)$ and the unimodal property of the function $m(u)$, we have $m\left(\alpha_{d^{\prime}-j-1}\right)<$ $m\left(\alpha_{d^{\prime}-j}\right)$. Note that if $\theta^{2}$ is irrational, then $m_{\theta}=m_{\theta^{\prime}}$ for some eigenvalue $\theta^{\prime}$ with $\theta^{\prime} \neq$ $\theta$. The assertion therefore follows from $m\left(\alpha_{d^{\prime}-j-1}\right)<m\left(\alpha_{d^{\prime}-j}\right)$ and Lemma 5.3.

Proposition 5.7. There does not exist a distance-regular graph $\Gamma$ with the quotient matrix $\boldsymbol{B}(k, d+1, c)$ for $d=11,16,24,25,26$.

Proof. By Lemma 5.6, we can estimate the number of irrational square eigenvalues $(k-1) \phi=\theta^{2}$ by checking $L\left(u_{d^{\prime}-j-1}^{(d-2)}, u_{d^{\prime}-j}^{(d-2)}\right)>1$ with a computer.

For $d=11$, the number of irrational $\phi$ is at most 1 for $k \geqslant 5$. For $k=3,4$, we can find an irreducible polynomial of degree at least 3 as a factor of $c^{\prime} P_{m-1, \epsilon}(z)+$ $k^{\prime} P_{m, \epsilon}(z)$ over $\mathbb{Q}$ for each pair $\left(c^{\prime}, k^{\prime}\right)$ with $0 \leqslant c^{\prime} \leqslant k^{\prime}-1$. We can find an irreducible polynomial of degree at least 3 or two irreducible polynomials of degree 2 as a factor of $c^{\prime} P_{m-1, \epsilon}(z)+k^{\prime} P_{m, \epsilon}(z)$ over $\mathrm{GF}(2)$ for each pair $\left(c^{\prime}, k^{\prime}\right) \in \mathrm{GF}(2) \times \mathrm{GF}(2)$.

For $d=16$, the number of irrational $\phi$ is at most 2 for $k \geqslant 3$. We can find an irreducible polynomial of degree at least 3 or three irreducible polynomials of degree 2 as a factor of $c^{\prime} P_{m-1, \epsilon}(z)+k^{\prime} P_{m, \epsilon}(z)$ over GF(3) for each pair $\left(c^{\prime}, k^{\prime}\right) \in \mathrm{GF}(3) \times \mathrm{GF}(3)$.

For $d=25$, the number of irrational $\phi$ is at most 2 for $k \geqslant 6$. For $k=3,4,5$, we can find an irreducible polynomial of degree at least 3 as a factor of $c^{\prime} P_{m-1, \epsilon}(z)+$ $k^{\prime} P_{m, \epsilon}(z)$ over $\mathbb{Q}$ for each pair $\left(c^{\prime}, k^{\prime}\right)$ with $0 \leqslant c^{\prime} \leqslant k^{\prime}-1$. We can find an irreducible polynomial of degree at least 3 or three irreducible polynomials of degree 2 as a factor of $c^{\prime} P_{m-1, \epsilon}(z)+k^{\prime} P_{m, \epsilon}(z)$ over $\mathrm{GF}(3)$ for each pair $\left(c^{\prime}, k^{\prime}\right) \in \mathrm{GF}(3) \times \mathrm{GF}(3)$.

For $d=24,26$, the number of irrational $\phi$ is at most 2 for $k \geqslant 4$. For $k=3$, we can find an irreducible polynomial of degree at least 3 as a factor of $c^{\prime} P_{m-1, \epsilon}(z)+k^{\prime} P_{m, \epsilon}(z)$ over $\mathbb{Q}$ for each $c^{\prime}$ with $0 \leqslant c^{\prime} \leqslant k^{\prime}-1$. We can find an irreducible polynomial of degree at least 3 or three irreducible polynomials of degree 2 as a factor of $c^{\prime} P_{m-1, \epsilon}(z)+$ $k^{\prime} P_{m, \epsilon}(z)$ over $\mathrm{GF}(3)$ for each pair $\left(c^{\prime}, k^{\prime}\right) \in \mathrm{GF}(3) \times \mathrm{GF}(3)$.

Theorem 5.1 follows from Table 7 and Propositions 5.2, 5.7.

\section{Conclusions}

In this paper, we studied $b(k, \theta)$, the maximum number of vertices in bipartite regular graph of valency $k$ whose second largest eigenvalue is at most $\theta$. Our results extend previous work from $[10,18,19,24,29]$. Our general bound for $b(k, \theta)$ is attained whenever there exists a bipartite distance-regular graph of valency $k$, second largest eigenvalue $\theta$, girth $g$ and diameter $d$ with $g \geqslant 2 d-2$. For $d=3$ and $g \geqslant 4$ all the point-block incidence graphs of symmetric designs give equality in our bound so the situation is well-understood. For $d \geqslant 4$ we only have the Van Lint-Schrijver geometry besides the generalized polygons. We believe that for $d \geqslant 5$ the only examples must have $c=1$ and are generalized polygons.

Acknowledgements. The authors thank Tatsuro Ito for providing useful information relating to Section 5 and the two anonymous referees for their useful comments and suggestions. 


\section{REFERENCES}

[1] Aida Abiad, Edwin R. van Dam, and Miquel Ángel Fiol, Some spectral and quasi-spectral characterizations of distance-regular graphs, J. Combin. Theory Ser. A 143 (2016), 1-18.

[2] Noga Alon, Eigenvalues and expanders, Combinatorica 6 (1986), no. 2, 83-96, Theory of computing (Singer Island, Fla., 1984).

[3] Noga Alon and Vitali D. Milman, $\lambda_{1}$, isoperimetric inequalities for graphs, and superconcentrators, J. Combin. Theory Ser. B 38 (1985), no. 1, 73-88.

[4] Eiichi Bannai and Tatsuro Ito, On the spectra of certain distance-regular graphs, J. Combin. Theory Ser. B 27 (1979), no. 3, 274-293.

[5] $\ldots$ On the spectra of certain distance-regular graphs. II, Quart. J. Math. Oxford Ser. (2) 32 (1981), no. 4, 389-411.

[6] _ Algebraic combinatorics. I: Association schemes, Mathematics lecture note series, The Benjamin/Cummings Publishing Co., Inc., Menlo Park, CA, 1984.

[7] Andries E. Brouwer, Arjeh M. Cohen, and Arnold Neumaier, Distance-regular graphs, Ergebnisse der Mathematik und ihrer Grenzgebiete (3) [Results in Mathematics and Related Areas (3)], vol. 18, Springer-Verlag, Berlin, 1989.

[8] Andries E. Brouwer and Willem H. Haemers, Spectra of graphs, Universitext, Springer, New York, 2012.

[9] Fan R. K. Chung, Diameters and eigenvalues, J. Amer. Math. Soc. 2 (1989), no. 2, 187-196.

[10] Sebastian M. Cioabă, Jack H. Koolen, Hiroshi Nozaki, and Jason R. Vermette, Maximizing the order of a regular graph of given valency and second eigenvalue, SIAM J. Discrete Math. 30 (2016), no. 3, 1509-1525.

[11] Henry Cohn and Abhinav Kumar, Universally optimal distribution of points on spheres, J. Amer. Math. Soc. 20 (2007), no. 1, 99-148.

[12] Robert Mark Damerell and Michael A. Georgiacodis, On the maximum diameter of a class of distance-regular graphs, Bull. London Math. Soc. 13 (1981), no. 4, 316-322.

[13] Giuliana Davidoff, Peter Sarnak, and Alain Valette, Elementary number theory, group theory, and Ramanujan graphs, London Mathematical Society Student Texts, vol. 55, Cambridge University Press, Cambridge, 2003.

[14] Frederick J. Fuglister, On generalized Moore geometries. I, Discrete Math. 67 (1987), no. 3, 249-258.

[15] _ On generalized Moore geometries. II, Discrete Math. 67 (1987), no. 3, 259-269.

[16] James Geronimus, On a set of polynomials, Ann. of Math. (2) 31 (1930), no. 4, 681-686.

[17] Lawrence A. Harris, Lagrange polynomials, reproducing kernels and cubature in two dimensions, J. Approx. Theory 195 (2015), 43-56.

[18] Tom Høholdt and Heeralal Janwa, Eigenvalues and expansion of bipartite graphs, Des. Codes Cryptogr. 65 (2012), no. 3, 259-273.

[19] Tom Høholdt and Jørn Justesen, On the sizes of expander graphs and minimum distances of graph codes, Discrete Math. 325 (2014), 38-46.

[20] Shlomo Hoory, Nathan Linial, and Avi Wigderson, Expander graphs and their applications, Bull. Amer. Math. Soc. (N.S.) 43 (2006), no. 4, 439-561.

[21] Akihito Hora and Nobuaki Obata, Quantum probability and spectral analysis of graphs, Theoretical and Mathematical Physics, Springer, Berlin, 2007, With a foreword by Luigi Accardi.

[22] Tamara Koledin and Zoran Stanić, Regular graphs with small second largest eigenvalue, Appl. Anal. Discrete Math. 7 (2013), no. 2, 235-249.

[23] , Reflexive bipartite regular graphs, Linear Algebra Appl. 442 (2014), 145-155.

[24] Wen-Ch'ing Winnie Li and Patrick Solé, Spectra of regular graphs and hypergraphs and orthogonal polynomials, European J. Combin. 17 (1996), no. 5, 461-477.

[25] Adam W. Marcus, Daniel A. Spielman, and Nikhil Srivastava, Interlacing families I: Bipartite Ramanujan graphs of all degrees, Ann. of Math. (2) 182 (2015), no. 1, 307-325.

[26] Mirka Miller and Jozef Sirán, Moore graphs and beyond: A survey of the degree/diameter problem, Electron. J. Combin. (2005), DS14-Dec (61 pages).

[27] Hiroshi Nozaki, Linear programming bounds for regular graphs, Graphs Combin. 31 (2015), no. $6,1973-1984$.

[28] Robert Singleton, On minimal graphs of maximum even girth, J. Combinatorial Theory 1 (1966), no. 3, 306-332.

[29] Yasuo Teranishi and Fumiko Yasuno, The second largest eigenvalues of regular bipartite graphs, Kyushu J. Math. 54 (2000), no. 1, 39-54. 
Sebastian M. Cioabă, University of Delaware, Department of Mathematical Sciences, Ewing Hall, Newark, DE 19716-2553, USA

E-mail : cioaba@udel.edu

Url : sites.udel.edu/cioaba

JACK H. Koolen, School of Mathematical Sciences, University of Science and Technology of China, Wen-Tsun Wu Key Laboratory of the Chinese Academy of Sciences, Hefei, Anhui, China

E-mail : koolen@ustc.edu.cn

Url : http://staff.ustc.edu.cn/ koolen/

Hiroshi Nozaki, Aichi University of Education, 1 Hirosawa, Igaya-cho, Kariya, Aichi 448-8542, Japan

E-mail : hnozaki@auecc.aichi-edu.ac.jp

Url : https://hnozaki.jimdo.com 